\title{
Error-proof programmable self-assembly of DNA-nanoparticle clusters
}

\author{
Nicholas A. Licata, Alexei V. Tkachenko \\ Department of Physics and Michigan Center for Theoretical Physics, \\ University of Michigan, 450 Church Str., Ann Arbor, Michigan 48109
}

\begin{abstract}
We study theoretically a new generic scheme of programmable self-assembly of nanoparticles into clusters of desired geometry. The problem is motivated by the feasibility of highly selective DNA-mediated interactions between colloidal particles. By analyzing both a simple generic model and a more realistic description of a DNA-colloidal system, we demonstrate that it is possible to suppress the glassy behavior of the system, and to make the self-assembly nearly error-proof. This regime requires a combination of stretchable interparticle linkers (e.g. sufficiently long DNA), and a soft repulsive potential. The jamming phase diagram and the error probability are computed for several types of clusters. The prospects for the experimental implementation of our scheme are also discussed.

PACS numbers: 81.16.Dn, 87.14.Gg, 36.40.Ei
\end{abstract}

\section{INTRODUCTION}

Over recent years, significant attention has been attracted to the possibility of nanotechnological applications of DNA 1], 2], [3], [4], [5]. Among the various proposals, one of the most interesting directions is the use of DNA-mediated interactions for programmable selfassembly of nanoparticle structures [6], [7], [8], [9]. Several schemes of such self-assembly have been studied both experimentally and theoretically. Their common theme is the use of colloidal particles functionalized with specially designed ssDNA (markers), whose sequence defines the particle type. In such systems, selective typedependent interactions can be introduced either by making the markers complementary to each other, or by using linker-DNA chains whose ends are complementary to particular marker sequences.

Recent theoretical studies have addressed the expected phase behavior [10], melting properties [11], and morphological diversity 12 of DNA-colloidal assemblies. In particular, there are indications that these techniques can be utilized for fabrication of photonic band gap materials [13], 14]. Despite significant experimental progress, the long-term potential of DNA-based self-assembly is far from being realized. For instance, most of the experimental studies of DNA-colloidal systems report random aggregation of the particles [15]. Some degree of structural control in these systems has been achieved, mostly by varying the relative sizes of particles, rather than by tuning the interactions [16], [17].

In the present paper, we take a broader view of programmable self-assembly. While this theoretical study is strongly motivated by the prospects of DNA-colloidal systems, our main objective is to address a more general question: how well can a desired structure be encoded by tunable interactions between its constituents? The particular model system on which we focus consists of distinguishable particles with individually controlled interactions between any pair of them. In the first stage of our study, we analyze a simplified yet generic version of such a system. All the particles have the same re- pulsive potential, while the attraction is introduced only between selected pairs of particles in the form of a springlike quadratic potential. This potential mimics the effect of a stretchable DNA molecule whose ends can selectively adsorb to the particular pair of particles. We then introduce a number of additional features which make the model a more realistic description of an actual DNAcolloidal system.

Our major result is that a combination of stretchable interparticle linkers (e.g. sufficiently long DNA), and a soft repulsive potential greatly reduces (or totally eliminates) the probability of self-assembling an undesired structure. The experimental prototype is a system of particles in a mixture of two types of DNA molecules which can selectively adsorb to the particle surface. The first type are DNA molecules with two "sticky" ends, i.e. both end sequences of the DNA are complementary to the particle marker sequence. With one end adsorbed to the particle surface, the remaining sticky end makes it possible to introduce an attractive interparticle potential between selected particle pairs. The second type are DNA molecules with one sticky end which adsorbs to the particle surface. These DNA strands give rise to a soft repulsive potential of entropic origin between all particle pairs.

There is a natural analogy between our problem and the folding of proteins where interactions between amino acids encode the overall structure. However, it should be emphasized that the task of programmable self-assembly in colloidal systems is even more demanding than protein folding: any intermediate metastable configuration has a much longer lifetime and therefore means a misfolding event. Because of this, we were looking for a self-assembly scenario which does not require thermally activated escape from a metastable configuration. This makes the problem additionally interesting from the theoretical perspective of "jamming", a phenomenon actively studied in the context of granular and colloidal systems. Our results can be interpreted in terms of a jamming-unjamming transition controlled by the interaction parameters.

It should be emphasized that the goal of this work 
is primarily conceptual, as opposed to providing a manual for the immediate experimental realization of ordered colloidal structures. Nevertheless, future experimental schemes will be forced to overcome obstacles presented by colloidal jamming. With this in mind, one of the most salient features of our model is the ability to smooth the energy landscape by tuning the interactions between particles.

The plan for the paper is as follows. In section II, we address the problem within a simplified generic model which mimics the nanoparticle system with stretchable DNA connections. An unexpected and very encouraging result of this study is that the misfolding (or jamming) in the model system can be completely avoided for a certain set of parameters. In section III the original model is adapted to a more realistic situation which incorporates the random character of the DNA-mediated interactions. In section IV we discuss the prospects for the future experimental implementation of our scheme. In section $\mathrm{V}$ we summarize the major results.

\section{BEADS AND SPRINGS MODEL}

Consider an isolated group of repulsive particles linked via a polymer spring to their desired nearest neighbours. We assume that the DNA marker sequence is the same for any two markers attached to the same particle, but different particles have different marker sequences (i.e. each particle has a unique code). In this case, the attraction between any two particles can be effectively switched on by adding DNA "linkers" whose ends are complementary to the corresponding marker sequences of the particles. As a first approach to the problem, we introduce a generic "Beads \& Springs" model which incorporates essential features of the DNA-nanoparticle system. The model system contains $N$ particles with pairwise (typeindependent) repulsive potential $U(r)$. In general, this repulsion may have a hard-core or soft-core behavior, or be a combination of the two. In order to model the DNAinduced type-dependent attraction, we introduce a harmonic potential (linear springs) which acts only between selected pairs of particles [18]. Thus, the model Hamiltonian has the following form:

$$
H=\frac{1}{2} \sum_{\alpha, \beta} \kappa J_{\alpha \beta}\left|\mathbf{r}_{\alpha}-\mathbf{r}_{\beta}\right|^{2}+U\left(\left|\mathbf{r}_{\alpha}-\mathbf{r}_{\beta}\right|\right)
$$

Here $\alpha, \beta$ are the particle indices, $\mathbf{r}_{\alpha}$ are their current positions, and $\kappa$ is the spring constant. The connectivity matrix element $J_{\alpha \beta}$ may be either 1 or 0 , depending on whether the two particles are connected by a spring, or not. Our goal is to program the desired spatial configuration by choosing an appropriate connectivity matrix $J_{\alpha \beta}$. A natural construction is to put $J_{\alpha \beta}=1$ for any pair of particles which must be nearest neighbors in the desired cluster, and not to connect the particles otherwise (i.e. put $J_{\alpha \beta}=0$ ). This construction assures that the target configuration is the ground state of the system.
Note that our problem is somewhat similar to that of heteropolymer folding. In that case, the selective interactions between monomers (e.g. amino acids in protein) are responsible for the coding of the spacial structure of a globule. Our major concern is whether the kinetics of the system will allow it to reach the ground state within a reasonable time. Unfortunately, since the Brownian motion of a typical nanoparticle is relatively slow (compared to molecular time scales), it is unrealistic to expect that our system will be able to find the target configuration by "hopping" between various metastable states, as in the case of protein folding. However, our case is different because the attractive force grows with distance, as opposed to the short-range nature of heteropolymer self-interactions. As we shall see below, this difference is essential, making it possible for the system to reach the ground state without stopping at any metastable configuration.

We have performed a molecular dynamics simulation of the above model by numerically integrating its Langevin equation:

$$
b^{-1} \dot{\mathbf{r}}_{\alpha}=-\nabla_{\alpha} H+\eta_{\alpha}
$$

Here $b$ is the particle mobility. The thermal noise has been artificially suppressed in this study (i.e. $\eta=0$ ). In other words, we have assumed the worst case scenario: once the system is trapped in a local energy minimum, it stays there indefinitely. The equations of motion were solved numerically by a first order Runge-Kutta method. First, we studied a system of $N=49$ distinguishable particles in $2 \mathrm{D}$, whose native configuration was a $7 \times 7$ square cluster (see Figure 1). Their initial positions were random, and the connectivity matrix was constructed according to the above nearest-neighbor rule.

First, we studied the case when $U(r)$ is a hard-core potential. More precisely, the repulsive force was given by a semi-linear form:

$$
f_{h c}(r)=-\frac{\partial U_{h c}(r)}{\partial r}=\kappa_{0}(d-r) \Theta(d-r)
$$

Here $\Theta$ is the unit step function, and $d$ is the diameter of the hard sphere. The parameter $\kappa_{0}$ determines the strength of the hard-core repulsion, and it does not affect the results, as long as $\kappa_{0} \gg \kappa$. In our simulations, we found that the hard sphere system eventually stops in a configuration definitely different from the desired one, a behavior which is well known in the context of granular and colloidal systems as "jamming" [19]. Remarkably, the jamming can be avoided when the hard-core repulsion is replaced by a soft-core potential:

$$
U_{s c}(r)=U_{0} \exp (-r / \lambda) .
$$

Here the decay length $\lambda$ is of the order of the equilibrium interparticle distance $r_{0}$. This indicates that the energy landscape can be made smooth by a combination of longrange selective attraction and soft-core repulsion. 

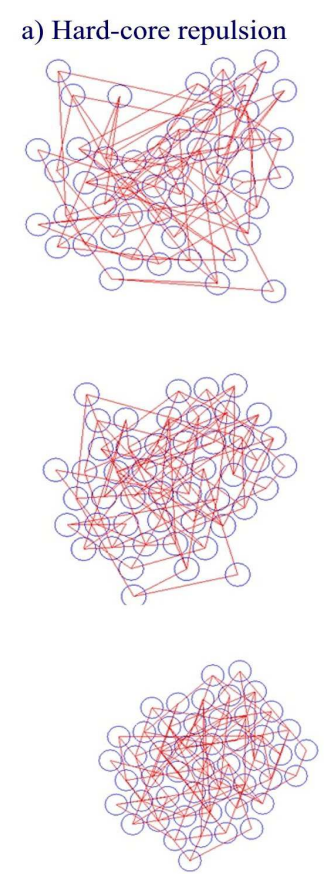

FIG. 1: (Color Online). Programmable self-assembly in 2D, studied within the Beads \& Springs model. 49 particles are all distinct and connected with linear springs to encode the desired configuration ( $7 \times 7$ square). The jamming behavior is observed for the case of hard spheres (a). However, the assembly of the target structure can be achieved if the repulsion is sufficiently "soft" (b).

The result is surprising and remarkably robust. In particular, in order to expand our finding to the 3D case, we studied the self-assembly of particles into tetrahedra of various sizes $(N=10,20,35)$. This time, the hard core interaction potential was superimposed with a soft shell repulsion, which makes the model more relevant for an actual DNA-colloidal system:

$$
U(r)=U_{h c}(r)+U_{s c}(r)
$$

After the system has fully relaxed, a geometric measure of the folding success is determined by comparing particle separations of the desired final state to those generated from a set of random initial conditions. Figure 2 shows the "jamming phase diagram" for these systems. To assign a point on the diagram to the correct folding regime, we required 100 consecutive successful folds. While this criterion can only give an upper bound on the jamming probability (which is approximately 1\%), an additional analysis gives strong evidence that the correct folding region of the diagram corresponds to zero probability of jamming.

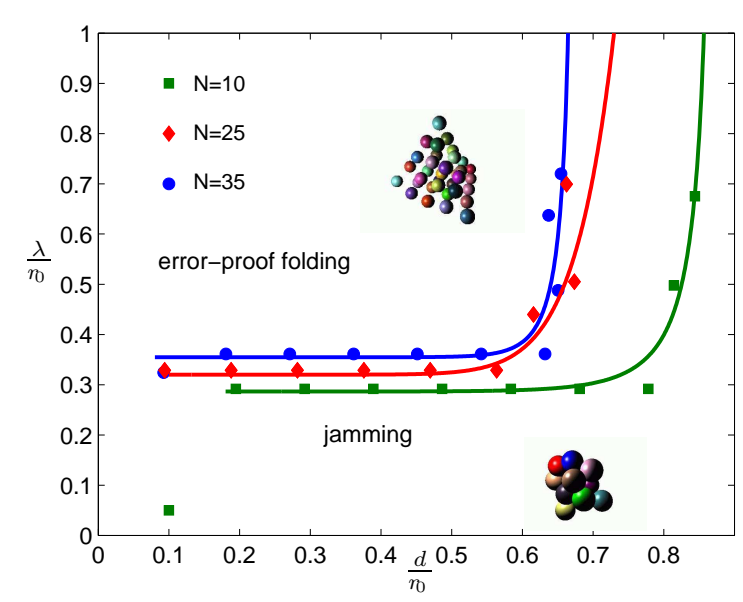

FIG. 2: (Color Online). "Jamming phase diagram" obtained for programmable self-assembly of tetrahedral clusters, within the Beads \& Springs model. The control parameters depend on the equilibrium interparticle distance $r_{o}$, the diameter of the hard sphere $d$, and the range of the soft-shell repulsion $\lambda$.

\section{SELF-ASSEMBLY IN DNA COLLOIDAL SYSTEMS}

As we have seen, the introduction of a soft-core repulsive potential $U_{s c}$ is crucial to a successful self-assembly proposal. In a real system, this repulsion can be generated e.g. by DNA or another water soluble polymer adsorbed to the particle surface. The mechanism is quite independent of the monomer chemistry, but for the sake of concreteness we will speak of the repulsion generated by DNA. Namely, we assume that a certain fraction of the DNA "arms" of the "octopus-like" particles are not terminated by a sticky end, and only play the role of a repulsive "buffer". When the polymer coverage is sufficiently low, the interparticle repulsion is primarily due to entropy loss of a chain squeezed between two particles. The characteristic length scale of this interaction is given by the radius of gyration of the "buffer" chain, $R_{g}$. The corresponding repulsive force $f_{s c}$ can be calculated exactly in the limit of relatively short buffer chains, $R_{g} \ll d$. The result of this calculation [12] can be adequately expressed in the following compact form:

$$
f_{s c}(r) \approx \frac{4 N R_{g} k_{B} T}{d(r-d)} \exp \left(-\frac{(r-d)^{2}}{2 R_{g}^{2}}\right)
$$

Here $N$ is the total number of buffer chains per particle. Note that even though this result is only valid for $R_{g} \ll d$, it correctly captures the Gaussian decay of the repulsive force expected for longer chains as well. Therefore, we expect the results to be at least qualitatively correct beyond the regime of short buffer chains.

In addition to the modified soft potential, we have to take into account the random character of the realistic DNA-mediated attraction. It originates from the 
fact that (1) the number of DNA "arms" of the original octopus-like particles will typically be determined by a random adsorption process, and (2) the fraction of the DNA chains recruited for linking a particular pair of particles is also random. In terms of our original model, this means that the "springs" will not have the same spring constant. If the individual linkers are modelled by Gaussian chains [20], the overall spring constant for a particular pair of connected particles is given by $\kappa_{\alpha \beta}=k_{B} T m_{\alpha \beta} / 2 R_{g}^{\prime 2}$, where $R_{g}^{\prime}$ is the radius of gyration of a single linker, and $m_{\alpha \beta}$ is the number of individual chains connecting the particles. We assume that this number obeys the generic Poisson distribution: $P(m)=\bar{m}^{m} e^{-\bar{m}} / m !$. As formulated, the model is cast as a system of coupled differential equations:

$\dot{\mathbf{r}}_{\alpha}=b \sum_{\beta}\left[-\frac{k_{B} T}{2 R_{g}^{\prime 2}} J_{\alpha \beta} m_{\alpha \beta} r_{\alpha \beta}+f_{h c}\left(r_{\alpha \beta}\right)+f_{s c}\left(r_{\alpha \beta}\right)\right] \hat{\mathbf{n}}_{\alpha \beta}$

Here $r_{\alpha \beta}=\left|\mathbf{r}_{\alpha}-\mathbf{r}_{\beta}\right|, \hat{\mathbf{n}}_{\alpha \beta}=\left(\mathbf{r}_{\alpha}-\mathbf{r}_{\beta}\right) / r_{\alpha \beta}$.

We have studied the behavior of the system as a function of two dimensionless parameters, one of which is the ratio of the buffer radius of gyration to the particle diameter, $R_{g} / d$. The other parameter characterizes the relative strength of the attractive and repulsive forces:

$$
\alpha=\frac{\bar{m}}{N}\left(\frac{R_{g}}{R_{g}^{\prime}}\right)
$$

Including the above modifications of the model, the essential result of the study is that the jamming probability can be drastically suppressed, similarly to the previous case. However, the jamming in this system cannot be eliminated completely. Instead of an actual "phase boundary", we have observed a sharp crossover to the regime of predominantly good folding, in which the error probability is suppressed to a modest level $\sim 10-20 \%$. Interestingly, the behavior is nearly independent of the energy parameter $\alpha$, which makes $R_{g} / d$ the only major control parameter. Figure 3 shows the error probability as a function of this geometric parameter for tetrahedral clusters of different sizes. As this plot indicates, the misfolding behavior gets suppressed as $R_{g} / d$ exceeds 1 .

As a further test of the robustness of the model, we consider a modified attractive potential which deviates from the linear Hooke's law. For larger forces we enter the Pincus regime[21], where the end-to-end extension of the polymer chain $r$ is related to the external tension $f \sim r^{\frac{3}{2}}$. This tension law incorporates the excluded volume interaction of individual linker DNA with themselves, which was not previously considered. Remarkably, the major result for error suppression carries over, as illustrated in figure 4

Our description has several limitations which are to be addressed in future work. In particular, our discussion is only applicable to the limit of modest coverage of particles with buffer chains. This case of weakly overlapping adsorbed chains (known as the "mushroom regime")

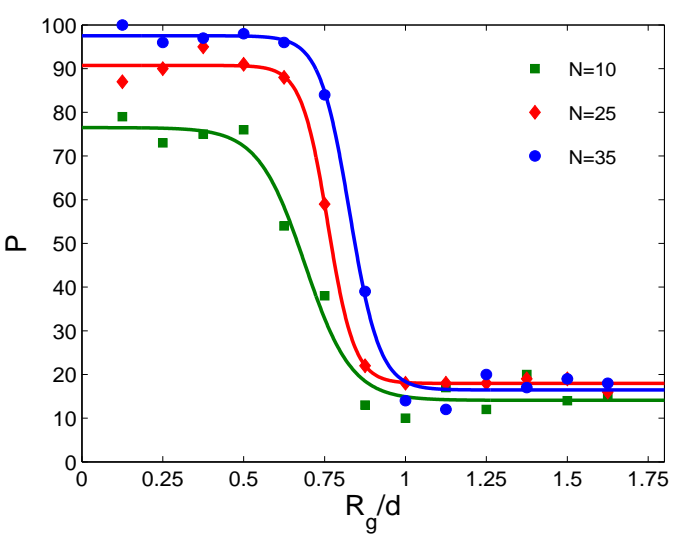

FIG. 3: (Color Online). Error probability $P$ as a function of aspect ratio $R_{g} / d$ for tetrahedral clusters with modified softpotential and realistic DNA-mediated attraction. Each data point on the misfolding profile represents 100 trials.

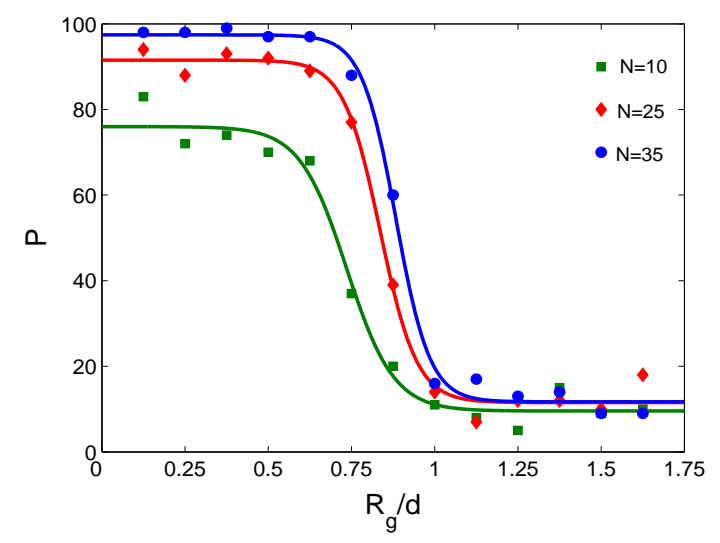

FIG. 4: (Color Online). Error probability $P$ as a function of aspect ratio $R_{g} / d$ for tetrahedral clusters in the Pincus regime. Each data point on the misfolding profile represents 100 trials.

is drastically different from the high-coverage "polymer brush" behavior 22]. Nevertheless, our major conclusions appear to be rather robust. The condition for error $\operatorname{suppression}\left(R_{g} / d \gtrsim 1\right)$ is the same in both the harmonic and Pincus regimes.

\section{DISCUSSION}

Previous studies have demonstrated an experimental implementation of self-assembly in a DNA-colloidal system [23], [24]. The approach in these studies differs from our vision of controllable self-assembly in two major ways: (1) because the "linker" DNA chains $(\sim 20 \mathrm{~nm})$ used are much shorter than the particle diameter $(\sim$ $1 \mu \mathrm{m})$, the particles behave as sticky spheres, and (2) there is little diversity in particle type, where the structures result from interactions of a one or two component 
system. We would like to discuss issues related to a future experimental implementation similar to the modified Beads \& Springs model.

In our molecular dynamics simulations we assumed that (1) the desired group of particles has already been localized in a small region of space, and that (2) the interparticle connections have already been made. There are a number of experimental challenges associated with implementing the self-assembly proposal of our simulations. In another manuscript 25] we provide a detailed discussion of the localization problem, which is the first major experimental intermediary.

After localization, the next step is to make the desired connections between particles within the cluster. To do so one can add short ssDNA with sequences $\bar{s}_{A} \bar{s}_{B}$ to link particles $A$ and $B$. The DNA marker sequence $s_{A}$ for particle $A$ is a sequence of nucleotides complementary to the $\bar{s}_{A}$ portion of the linker sequence $\bar{s}_{A} \bar{s}_{B}$. The hydrogen bonding of complementary nucleotides forms base pairs which join both marker strands to the linker, creating a DNA bridge between the two particles. After the interparticle links are formed, they should be made permanent by ligation. Since the spring constants of the above dsDNA chains are too small to drive the selfassembly of a desired cluster, we propose to melt them either by changing the temperature or $\mathrm{pH}$. As a result, the dsDNA links will be turned into ssDNA with a much higher effective spring constant (due to the shorter persistence length). This will trigger the self-assembly scenario similar to the one discussed within the Beads \& Springs model. Note that DNA entanglements may be effectively eliminated if the procedure is done in the presence of DNA Topoisomerases.

\section{CONCLUSION}

In conclusion, we presented a model of DNAcolloidal self-assembly which exhibits a tunable jamming- unjamming transition. The combination of a soft-core repulsion with a type-dependent long range attraction provides a natural funneling of the energy landscape to the ground state configuration. This is to be contrasted with the case of protein folding, where under physiological conditions the interactions between amino acids are screened to several angstroms. Because this lengthscale is much shorter than the spatial extent of the native structure, large regions of the energy landscape are flat, which prohibits formation of the native state on the basis of funneling alone. As a result the folding rate is necessarily limited by the diffusion of amino acid segments looking for their desired nearest neighbors [26], 27]. The fact that the potential in the DNA-colloidal system is long ranged is essential, allowing us to avoid the pitfall of slow particle diffusion.

Within the Beads and Springs Model, we obtained the jamming phase diagram for several modest sized tetrahedral clusters. We identified a regime of parameter space with error-proof folding, and demonstrated the importance of introducing a soft-core repulsion. The original model was then adapted to include several features of realistic DNA-mediated interactions. Although the jamming cannot be completely eliminated in the modified system, we identified a regime of predominantly good folding, and calculated the error probability for tetrahedral clusters. The jamming behavior is determined by a single geometric parameter $R_{g} / d$. We concluded by discussing prospects for an experimental implementation of our self-assembly scheme.

\section{ACKNOWLEDGEMENTS}

This work was supported by the ACS Petroleum Research Fund (grant PRF \#44181-AC10), and by the Michigan Center for Theoretical Physics (award \# MCTP 06-03).
[1] Seeman, N. C. Angew. Chem. Int. Ed. 1998, 37, 32203238.

[2] Ball, P. Nature 2001, 413, 667-668.

[3] Braun, E.; Eichen, Y.; Sivan, U.; Ben-Yoseph, G. Nature 1998, 391, 775-778.

[4] Wengel, J. Org. Biomol. Chem 2004, 2, 277-280.

[5] Dwyer, C.; Johri, V.; Cheung, M.; Patwardhan, J.; Lebeck, A.; Sor, D. Nanotechnology 2004, 15, 1240-1245.

[6] Mirkin, C. A.; Letsinger, R. L.; Mucic, R. C.; Storhoff, J. J. Nature 1996, 382, 607 - 609.

[7] Seeman, N. C. Nature 2003, 421, 427-431.

[8] Winfree, E.; Liu, F.; Wenzler, L. A.; Seeman, N. C. Nature 1998, 394, 539-544.

[9] Cobbe, S.; Connolly, S.; Ryan, D.; Nagle, L.; Eritja, R.; Fitzmaurice, D. J. Phys. Chem. B 2003, 107, 470-477.

[10] Lukatsky, D.; Frenkel, D. Physical Review Letters 2004, 92,068302 .
[11] Jin, R.; Wu, G.; Li, Z.; Mirkin, C. A.; Schatz, G. C. JACS 2003, 125, 1643-1654.

[12] Tkachenko, A. V. Physical Review Letters 2002, 89, 148303.

[13] Fan, S.; Villeneuve, P. R.; Joannopoulos, J. D. Phys. Rev. B 1996, 54, 11245-11251.

[14] Sievenpiper, D. F.; Sickmiller, M. E.; Yablonovitch, E. Phys. Rev. Lett. 1996, 76, 2480-2483.

[15] Sun, Y.; Harris, N. C.; Kiang, C.-H. Physica A 2005, $354,1-9$.

[16] Milam, V. T.; Hiddessen, A. L.; Crocker, J. C.; Graves, D. J.; Hammer, D. A. Langmuir 2003, 19, 10317-10323.

[17] Soto, C. M.; Srinivasan, A.; Ratna, B. R. JACS 2002, 124, 8508-8509.

[18] Jones, R. A. L. Soft Condensed Matter; Oxford University Press: Great Clarendon Street, Oxford OX2 6DP, 
U.K., 2003.

[19] Segre, P.; Prasad, V.; Schofield, A.; Weitz, D. Physical Review Letters 2001, 86, 6042-6045.

[20] Grosberg, A. Y.; Khokhlov, A. Statistical Physics of Macromolecules; AIP, New York: AIP Press, American Institute of Physics, 500 Sunnyside Boulevard, Woodbury, NY 11797-2999, 1994.

[21] Pincus, P. Macromolecules 1976, 9, 386-388.

[22] de Gennes, P. Scaling Concepts of Polymer Physics; Cornell University Press, Ithaca, NY: Ithaca, NY, 1979.
[23] Valignat, M.-P.; Theodoly, O.; Crocker, J. C.; Russel, W. B.; Chaikin, P. M. PNAS 2005, 102, 4225-4229.

[24] Biancaniello, P. L.; Kim, A. J.; Crocker, J. C. PRL 2005, 94, 058302.

[25] Licata, N. A.; Tkachenko, A. V. arXiv:condmat/0606397 v1 2006, .

[26] Onuchic, J. N.; Luthey-Schulten, Z.; Wolynes, P. G. Annu. Rev. Phys. Chem. 1997, 48, 545-600.

[27] McLeish, T. C. B. Biophys. J. 2005, 88, 172-183. 